Punjab University Journal of Mathematics

(ISSN 1016-2526)

Vol. 53(3)(2021) pp. 1-7

\title{
Some Results of Self-Maps in PU-Algebra
}

\author{
Dawood Khan \\ Department of Mathematics, \\ University of Balochistan, Quetta 87300,Pakistan \\ Email: dawooddawood601@gmail.com \\ Abdul Rehman \\ Department of Mathematics, \\ University of Balochistan, Quetta87300,Pakistan \\ Email: abdul_maths@yahoo.com \\ Saleem Iqbal \\ Department of Mathematics, \\ University of Balochistan, Quetta87300,Pakistan \\ Email: saleemiqbal81@yahoo.com \\ Naveed Sheikh \\ Department of Mathematics, \\ University of Balochistan, Quetta87300,Pakistan \\ Email: naveed_maths@hotmail.com
}

Received: 05 August, 2020 / Accepted: 20 January, 2021 / Published online: 15,March, 2021

Abstract.: In this manuscript we examine the concept of left and right self-maps in PU-Algebra and explore some further interesting prperties to PU-Algebra.We prove that $\underline{R}_{x}^{2}$ is idempotent, isotonic and endomorphic. We determine the condition for which the composition of $\underline{L}_{\alpha}$ and $\underline{R}_{b}$ is equivalent to $\underline{R}_{o}$. We prove that under what condition $\underline{L}_{x}^{n}$ is an endomorphism. We define the $\operatorname{Ker}\left(R_{x}^{2}\right)$ and show that it is a subalgebra and ideal of PU-Algebra. We also prove that the $\operatorname{Fix}\left(\underline{R}_{x}^{2}\right)$ is a subalgebra and ideal of PU-Algebra.

AMS (MOS) Subject Classification Codes: 06Fxx; 06F35

Key Words: PU-Algebra, Left self-maps, Right Self-maps.

\section{INTRODUCTION}

In 1966, Imai and Iseki [2] introduced two classes of abstract algebra: BCK-algebras and BCI-algebras. It is known that the class BCK-algebras is a proper sub class of the 
class of BCI-algebras. In [1], Hu and Li introduced a wide class of abstract algebras: $\mathrm{BCH}$-algebras. They are shown that the class of BCI-algebras is a proper sub class of the class of BCH-algebras. In [5], Neggers and Kim introduced the Notion of d-algebra, which is a generalization of BCK-algebras and investigated a relation between d-algebras and BCK-algebras. Neggers et al.[6] introduced the notion of Q-algebras, which is the generalization of $\mathrm{BCH} / \mathrm{BCI} / \mathrm{BCK}$-algebras. Magalai and Tamilarasi [3] introduced the notion of a TM-algebra which is a generalization of $\mathrm{BCK} / \mathrm{BCI} / \mathrm{BCH}$-algebras and several results are presented. The foundation of the concept of PU-algebra was laid down by the mathematicians Mostafa et al. in their pioneering paper [4]. PU-algebra is a dual for TM-algebra. It lies in a category of type $(2,0)$ algebras. $(2,0)$ types algebras consist of two operations one is binary opertaion and other is nullary operation. Algebras like BCK,BCI,BCH,TM etc. are all the type $(2,0)$ algebras. Their theory about PU-algebra and related ideas and properties are nowadays utilized extensively in different areas of science like artificial intelligence, information sciences, cybernetics and computer sciences. The algebras like PU-algebras, BCK-algebras, and BCI-algebras have been inspired by two considerations, one based on classical and non-classical propositional calculi of Meredith and other based on set theory [2]. The concept of ideal theory of PU-algebra plays a fundamental role in the evolution of PU-Algebra, was first introduced by Mostafa et al. in [4] while the notion of PU-homomorphism was also first defined by Mostafa et al. in [4]. They have stated and proved some fundamental properties of it. Moreover, they have given the concept of right self-maps, left self-maps, weak right self-maps and weak left self-maps and investigated some of its properties. In this paper we shall further investigate these maps and determine further interesting properties.

We have no doubt that the research along this line can be kept up, and indeed, some results in this manuscript have already made up a foundation for further exploration concerning the further progression of left and right self-maps as well as to insert these results in exploring the properties of weak right self-maps and weak left self-maps and their applications in other disciplines of algebra.

\section{Preliminaries}

This section consists of some preliminary definitions and basic facts about PU-algebra which are useful in the proofs of our results. Throughout this research work we denote the PU-algebra always by $Z$ without any specification. Here we only mention those concepts of PU-algebra which are necessary for our treatment.

Definition 2.1. [4] PU-algebra $(Z, *, 0)$ is a class of the type $(2,0)$ algebras satisfying the $\left(P_{1}\right)$ and $\left(P_{2}\right)$ conditions for all $a, b, c \in Z$, where

$\left(P_{1}\right) \quad 0 * a=a . \quad\left(P_{2}\right) \quad(a * c) *(b * c)=b * a$.

While the binary relation $\leq$ on $Z$ is defined as $a \leq b \Leftrightarrow b * a=0$.

Proposition 2.2. [4] The following results hold in any PU-algebra $(Z, *, 0)$ for all $a, b, c \epsilon$ $Z$.

$\left(P_{3}\right) \quad a * a=0$.

$\left(P_{4}\right) \quad(a * c) * c=a$.

$\left(P_{5}\right) \quad a *(b * c)=b *(a * c)$.

$\left(P_{6}\right) \quad a *(b * a)=b * 0$.

$\left(P_{7}\right) \quad(a * b) * 0=b * a$. 
$\left(P_{8}\right) \quad$ if $a \leq b$ then $a * 0=b * 0$.

$\left(P_{9}\right) \quad(a * b) * 0=(a * c) *(b * c)$.

$\left(P_{10}\right) \quad(a * b) \leq c \Leftrightarrow c * b \leq a$.

$\left(P_{11}\right) \quad a \leq b \Leftrightarrow(b * c) \leq(a * c)$.

$\left(P_{12}\right) \quad$ The following three results are similar in $(Z, *, 0)$.

(1): $b=c$. (2): $b * a=c * a$. (3): $a * b=a * c$.

$\left(P_{13}\right) \quad$ Both (left and right) cancellation properties hold in $(Z, *, 0)$.

Proposition 2.3. [4] In PU-algebra $(Z, *, 0)$ the conditions $\left(P_{14}\right)$ and $\left(P_{15}\right)$ hold for all $a, b, c \in Z$. Where

$\left(P_{14}\right) \quad(c * a) *(c * b)=a * b . \quad\left(P_{15}\right) \quad(a * b) * c=(c * b) * a$.

Definition 2.4. [4] Let $(Z, *, 0)$ is a PU-algebra then for any fixed $x \in Z$ the mapping $\underline{R}_{x}: Z \rightarrow Z$, defined by $\underline{R}_{x}(a)=a * x, \forall a \epsilon Z$, is said to be right self- map of $Z$. Similarly the mapping $\underline{L}_{x}: Z \rightarrow Z$, defined by $\underline{L}_{x}(a)=x * a, \forall a \epsilon Z$, is said to be left self- map of $Z$.

Definition 2.5. [4] Let $Z_{s}$ is a non-vacuous subset of $(Z, *, 0)$ then $Z_{s}$ is called PU- subalgebra of $Z$ if $a * b \in Z_{s}$ whenever $a, b \in Z_{s}$

Definition 2.6. [4] Let $Z_{d}$ is a non-vacuous subset of $(Z, *, 0)$ is said to be ideal of $Z$ if it satisfies the $\left(P_{16}\right)$ and $\left(P_{17}\right)$ conditions for any $a, b \in Z$, where

$\left(P_{16}\right) \quad 0 \epsilon Z_{d} . \quad\left(P_{17}\right) \quad a * b \epsilon Z_{d}$ and $a \epsilon Z_{d} \Rightarrow b \in Z_{d}$.

\section{RIGHT SELF-MAPS IN PU-ALGEBRA}

In this section we prove the results of right self-maps which are useful in proving the theorems in section-4.

Proposition 3.1. Let $Z$ be a PU-algebra then the following results are true for all $a, b \in Z$.

$\left(P_{a}\right) \quad$ If $\underline{R}_{x}(0)=0$ then $x=0, \quad \forall x \in Z$.

$\left(P_{b}\right) \quad$ If $\underline{R}_{x}(b) * \underline{R}_{x}(a)=\underline{R}_{a * b}(0) \quad \forall x \in Z$.

$\left(P_{c}\right) \quad \underline{R}_{x}^{2}$ is idempotent i.e. $R_{x}^{2} \circ R_{x}^{2}=R_{x}^{2} \quad \forall x \in Z$.

$\left(P_{d}\right) \quad \underline{R}_{x}^{2}$ is an endomorphism $\forall x \in Z$.

$\left(P_{e}\right) \quad \underline{R}_{x}^{2}(a * b)=\underline{R}_{x}(b) * \underline{R}_{x}(a) . \quad \forall x \in Z$.

$\left(P_{f}\right) \quad \underline{R}_{x}^{2}(a * b)=\underline{R}_{x}^{2}(a) * b=a * \underline{R}_{x}^{2}(b) . \quad \forall x \in Z$.

$\left(P_{g}\right) \quad a \leq b \Rightarrow\left\{\begin{array}{lll}\underline{R}_{x}^{2}(a) \leq \underline{R}_{x}^{2}(b) & \text { if } n \text { is even natural number } & \forall x \in Z . \\ \underline{R}_{x}^{2}(b) \leq \underline{R}_{x}^{2}(a), & \text { if } n \text { is odd natural number } & \forall x \in Z .\end{array}\right.$

$\left(P_{h}\right)$ For every natural number $n, \underline{R}_{x}^{n}= \begin{cases}\underline{R}_{x} & \text { if } n \text { is odd } \quad \forall x \in Z . \\ I, & \text { if } n \text { is even } \quad \forall x \in Z .\end{cases}$

Here ${ }^{\prime} I$ is identity map.

$\left(P_{i}\right) \quad \underline{R}_{x}^{n}(a) * \underline{R}_{x}^{n}(b)=\left\{\begin{array}{lll}a * b, & \text { if } n \text { is even natural number } & \forall x \in Z . \\ b * a, & \text { if } n \text { is odd natural number } & \forall x \in Z .\end{array}\right.$

Proof.

$\left(P_{a}\right): \quad$ If $\underline{R}_{x}(0)=0$, then $0 * x=0$. From proposition $\left(P_{3}\right)$, we have $x * x=0$, so $x * x=0 * x$. By $\left(P_{13}\right)$ both (left and right) cancellation properties hold in $(Z, *, 0)$, so $x=0$. 
$\left(P_{b}\right):$ Since $\underline{R}_{x}(b) * \underline{R}_{x}(a)=(b * x) *(a * x)$. From proposition $\left(P_{2}\right)$, we have $\underline{R}_{x}(b) * \underline{R}_{x}(a)=a * b$. By $\left(P_{1}\right)$, we have $\underline{R}_{x}(b) * \underline{R}_{x}(a)=0 *(a * b)$, so $\underline{R}_{x}(b) * \underline{R}_{x}(a)=$ $\underline{R}_{a * b}(0)$.

$\left(P_{c}\right)$ : Since $\left(R_{x}^{2} \circ R_{x}^{2}\right)(a)=R_{x}^{2}\left(R_{x}^{2}(a)\right)$, so $\left(R_{x}^{2} \circ R_{x}^{2}\right)(a)=R_{x}^{2}(((a * x) * x))$. From proposition $\left(P_{4}\right)$, we have $\left(R_{x}^{2} \circ R_{x}^{2}\right)(a)=R_{x}^{2}(a)$.

$\left(P_{d}\right): \underline{R}_{x}^{2}(a * b)=((a * b) * x) * x$. From proposition $\left(P_{4}\right)$, we have $\underline{R}_{x}^{2}(a * b)=a * b$. By $\left(P_{4}\right)$, we have $\underline{R}_{x}^{2}(a * b)=[(a * x) * x] *[(b * x) * x]$, so $\underline{R}_{x}^{2}(a * b)=\underline{R}_{x}^{2}(a) * \underline{R}_{x}^{2}(b)$. Hence $\underline{R}_{x}^{2}$ is an endomorphism

$\left(P_{e}\right): \underline{R}_{x}^{2}(a * b)=((a * b) * x) * x$. From proposition $\left(P_{4}\right)$, we have $\underline{R}_{x}^{2}(a * b)=a * b$.

By $\left(P_{4}\right)$, we have $\underline{R}_{x}^{2}(a * b)=((a * x) * x) * b$. By $\left(P_{15}\right)$, we get $\underline{R}_{x}^{2}(a * b)=(b * x) *(a * x)$, so $\underline{R}_{x}^{2}(a * b)=\underline{R}_{x}(b) * \underline{R}_{x}(a)$.

$\left(P_{f}\right): \underline{R}_{x}^{2}(a * b)=((a * b) * x) * x$. From proposition $\left(P_{4}\right)$, we have $\underline{R}_{x}^{2}(a * b)=a * b$. Again by $\left(P_{4}\right)$, we get $\underline{R}_{x}^{2}(a * b)=((a * x) * x) * b$, so $\underline{R}_{x}^{2}(a * b)=\underline{R}_{x}^{2}(a) * b$.

Similarly $\underline{R}_{x}^{2}(a * b)=a *((b * x) * x)=a * \underline{R}_{x}^{2}(b)$.

Hence $\underline{R}_{x}^{2}(a * b)=\underline{R}_{x}^{2}(a) * b=a * \underline{R}_{x}^{2}(b)$.

$\left(P_{g}\right):$ If $a \leq b$, then by $\left(P_{11}\right)$,we have $b * x \leq a * x$, so $\underline{R}_{x}(b) \leq \underline{R}_{x}(a)$.

if we have $b * x \leq a * x$, then again by $\left(P_{11}\right)$, we have $(a * x) * x \leq(b * x) * x$, so $\underline{R}_{x}^{2}(a) \leq \underline{R}_{x}^{2}(b)$.

Next if we have $(a * x) * x \leq(b * x) * x$, then again by $\left(P_{11}\right)$ we get $((b * x) *) * x \leq((a * x) *) * x$, so $\underline{R}_{x}^{3}(b) \leq \underline{R}_{x}^{3}(a)$.

Continuing in the same way we reach to a conclusion that $a \leq b \Rightarrow\left\{\begin{array}{lll}\underline{R}_{x}^{n}(a) \leq \underline{R}_{x}^{n}(b), & \text { if } n \text { is even natural number } & \forall x \in Z . \\ \underline{R}_{x}^{n}(b) \leq \underline{R}_{x}^{n}(a), & \text { if } n \text { is odd natural number } & \forall x \in Z .\end{array}\right.$

$\left(P_{h}\right)$ : For $n=1$, we have $\underline{R}_{x}^{1}(a)=a * x \Rightarrow \underline{R}_{x}^{1}(a)=\underline{R}_{x}(a) \Rightarrow \underline{R}_{x}^{1}=\underline{R}_{x}$

For $n=2$, we get $\underline{R}_{x}^{2}(a)=(a * x) * x$. From proposition $\left(P_{4}\right)$, we have $\underline{R}_{x}^{2}(a)=a$, so $\underline{R}_{x}^{2}(a)=I$.

For $n=3$, we have

$\underline{R}_{x}^{3}(a)=((a * x) * x) * x$. From proposition $\left(P_{4}\right)$, we get $\underline{R}_{x}^{3}(a)=a * x \Rightarrow \underline{R}_{x}=\underline{R}_{x}^{3}$.

For $n=4$, we have

$\underline{R}_{x}^{4}(a)=(((a * x) * x) * x) * x$. From proposition $\left(P_{4}\right)$, we have $\underline{R}_{x}^{4}(a)=(a * x) * x$.

Again by $\left(P_{4}\right)$, we get $\underline{R}_{x}^{4}(a)=a$, so $\underline{R}_{x}^{4}(a)=I(a)$.

Continuing in the same way we reach to a conclusion that if ' $n$ ' is positive odd integer then $\underline{R}_{x}^{n}=\underline{R}_{x}$. And if ' $n^{\prime}$ is positive even integer then $\underline{R}_{x}^{n}=I$.

$\left(P_{i}\right)$ : For $n=1$, we have $\underline{R}_{x}^{1}(a) * \underline{R}_{x}^{1}(b)=(a * x) *(b * x)$. From proposition $\left(P_{2}\right)$, we have $\underline{R}_{x}^{1}(a) * \underline{R}_{x}^{1}(b)=b * a$.

For $n=2$, we have $\underline{R}_{x}^{2}(a) * \underline{R}_{x}^{2}(b)=((a * x) * x) *((b * x) * x)$. From proposition $\left(P_{2}\right)$, we have $\underline{R}_{x}^{2}(a) * \underline{R}_{x}^{2}(b)=(b * x) *(a * x)$. Again by $\left(P_{2}\right)$, we have $\underline{R}_{x}^{2}(a) * \underline{R}_{x}^{2}(b)=a * b$. Continuing in the same way we reach to a conclusion that if ' $n$ ' is positive odd integer then $\underline{R}_{x}^{n}(a) * \underline{R}_{x}^{n}(b)=b * a$. And if ' $n^{\prime}$ is positive even integer then $\underline{R}_{x}^{n}(a) * \underline{R}_{x}^{n}(b)=a * b$. 


\section{LEFT SELF-MAPS IN PU-ALGEBRAS}

In this section we prove the results of left self-maps which are useful in proving the theorems related to endomorphism, subalgebras and ideals in PU-algebras.

Proposition 4.1. Let $Z$ be a PU-algebra then the following results are true for all $a, b \in Z$. $\left(P_{j}\right) \quad \underline{L}_{o}$ is the identity map.

$\left(P_{k}\right) \quad \underline{L}_{x}(a) * \underline{L}_{x}(b)=\underline{L}_{a}(b)$

$\forall x \in Z$.

$\left(P_{l}\right) \quad a * \underline{L}_{x}^{2}(b)=\underline{L}_{x}^{2}(a * b)$

$\forall x \in Z$.

$\left(P_{m}\right) \quad \underline{L}_{x}^{2}(a) * \underline{L}_{x}^{2}(b)=\underline{L}_{x}(a) * \underline{L}_{x}(b)$,

$\left(P_{n}\right) \quad$ For all natural numbers $n, \underline{L}_{x}^{n}(a * b)=a * \underline{L}_{x}^{n}(b), \quad \forall x \in Z$.

$\left(P_{o}\right) \quad$ For all natural numbers $n, \underline{L}_{x}^{n}(a) * \underline{L}_{x}^{n}(b)=a * b, \quad \forall x \in Z$.

Proof.

$\left(P_{j}\right): \underline{L}_{o}(a)=0 * a$. From proposition $\left(P_{1}\right)$, we have $\underline{L}_{o}(a)=a$, so $\underline{L}_{o}(a)=I(a)$, or $\underline{L}_{o}=I, \forall a \epsilon Z$.

$\left(P_{k}\right): \underline{L}_{x}(a) * \underline{L}_{x}(b)=(x * a) *(x * b)$. From proposition $\left(P_{14}\right)$, we have $\underline{L}_{x}(a) * \underline{L}_{x}(b)=$ $a * b=\underline{L}_{a}(b)$.

$\left(P_{l}\right): a * \underline{L}_{x}^{2}(b)=a *(x *(x * b))$. From proposition $\left(P_{5}\right)$, we have $a * \underline{L}_{x}^{2}(b)=x *(a *(x * b))$. Again by $\left(P_{5}\right)$,we get $a * \underline{L}_{x}^{2}(b)=x *(x *(a * b))$, so $a * \underline{L}_{x}^{2}(b)=\underline{L}_{x}^{2}(a * b)$.

$\left(P_{m}\right): \underline{L}_{x}^{2}(a) * \underline{L}_{x}^{2}(b)=[x *(x * a)] *[x *(x * b)]$. From proposition $\left(P_{14}\right)$, we get $\underline{L}_{x}^{2}(a) * \underline{L}_{x}^{2}(b)=(x * a) *(x * b)$, so $\underline{L}_{x}^{2}(a) * \underline{L}_{x}^{2}(b)=\underline{L}_{x}(a) * \underline{L}_{x}(b)$.

$\left(P_{n}\right): \underline{L}_{x}^{n}(a * b)=a * \underline{L}_{x}^{n}(b)$.

We prove this by using the principle of mathematical induction.

So for $n=1$, we have $\underline{L}_{x}(a * b)=a * \underline{L}_{x}(b)$. From proposition $\left(P_{5}\right)$, we get $\underline{L}_{x}(a * b)=$ $a *(x * b))$, so $\underline{L}_{x}(a * b)=a * \underline{L}_{x}(b)$. Therefore the given statement is true for $n=1$. Next we assume that the given statement is true for $n=k$, such that $\underline{L}_{x}^{k}(a * b)=a * \underline{L}_{x}^{k}(b)$.

Then $\underline{L}_{x}^{k+1}(a * b)=\underline{L}_{x}\left(\underline{L}_{x}^{k}(a * b)\right)$,

$\Rightarrow \underline{L}_{x}^{k+1}(a * b)=\underline{L}_{x}\left(a * \underline{L}_{x}^{k}(b)\right)=x *\left(a * \underline{L}_{x}^{k}(b)\right)$.

From $\left(P_{5}\right)$, we get $\underline{L}_{x}^{k+1}(a * b)=a *\left(x * \underline{L}_{x}^{k}(b)\right)$, so $\underline{L}_{x}^{k+1}(a * b)=a * \underline{L}_{x}\left(\underline{L}_{x}^{k}(b)\right)=$ $a * \underline{L}_{x}^{k+1}(b)$,

Hence the given statement is true for all natural numbers.

$\left(P_{o}\right): \underline{L}_{x}^{n}(a) * \underline{L}_{x}^{n}(b)=a * b$.

We prove this by using the principle of mathematical induction.

So for $n=1$, we have $\underline{L}_{x}(a) * \underline{L}_{x}(b)=(x * a) *(x * b)$

From proposition $\left(P_{14}\right)$,we get $\underline{L}_{x}(a) * \underline{L}_{x}(b)=a * b$, so the given statement is true for $n=1$.

Now next we assume that the given statement is true for $n=k$, such that $\underline{L}_{x}^{k}(a) * \underline{L}_{x}^{k}(b)=a * b$. Then $\underline{L}_{x}^{k+1}(a) * \underline{L}_{x}^{k+1}(b)=\left(x * \underline{L}_{x}^{k}(a)\right) *\left(x * \underline{L}_{x}^{k}(b)\right)$.

Again by $\left(P_{14}\right)$,we get $\underline{L}_{x}^{k+1}(a) * \underline{L}_{x}^{k+1}(b)=\underline{L}_{x}^{k}(a) * \underline{L}_{x}^{k}(b)$, so $\underline{L}_{x}^{k+1}(a) * \underline{L}_{x}^{k+1}(b)=a * b$.

Hence the given statement is true for all natural numbers.

Proposition 4.2. Let $Z$ be a PU-Algebra then the following results are true for all $x \in Z$.

$\left(P_{p}\right) \quad \underline{R}_{x}^{2}$ is isotonic, i.e. $a \leq b \Rightarrow \underline{R}_{x}^{2}(a) \leq \underline{R}_{x}^{2}(b), \forall a, b \in Z$.

$\left(P_{q}\right) \quad \underline{L}_{x} \circ \underline{R}_{x}=\underline{R}_{o}, \forall x \in Z$.

$\left(P_{r}\right) \quad \underline{R}_{x}^{2}(b)=0 \Leftrightarrow \underline{L}_{b}(x)=x, \forall \quad b \in Z$.

Proof.

$\left(P_{p}\right)$ : Let $a \leq b$. From proposition $\left(P_{11}\right)$, we have $b * x \leq a * x$. 
Again from $\left(P_{11}\right)$, we have $(a * x) * x \leq(b * x) * x$, so $\underline{R}_{x}^{2}(a) \leq \underline{R}_{x}^{2}(b)$.

Hence $a \leq b \Rightarrow \underline{R}_{x}^{2}(a) \leq \underline{R}_{x}^{2}(b), \forall \quad a, b \in Z$.

$\left.\left(P_{q}\right):\left(\underline{L}_{x} \circ \underline{R}_{x}\right)(b)=\underline{L}_{x}\left(\underline{R}_{x}\right)(b)\right)=x *(b * x), \forall \quad b \in Z$.

From proposition $\left(P_{6}\right)$, we get $\left(\underline{L}_{x} \circ \underline{R}_{x}\right)(b)=b * 0=\underline{R}_{o}(b)$, so $\underline{L}_{x} \circ \underline{R}_{x}=\underline{R}_{o}$.

$\left(P_{r}\right): \underline{R}_{x}^{2}(b)=0$.Then $(b * x) * x=0$. From proposition $\left(P_{4}\right)$, we have $b=0$.

By $\left(P_{12}\right), b=0$, is identical to $b * x=0 * x$.

By $\left(P_{1}\right)$, we get $b * x=x$, so $\underline{L}_{b}(x)=x, \forall \quad b \in Z$.

Conversely, Let $\underline{L}_{b}(x)=x$, so $b * x=x$.

From proposition $\left(P_{12}\right)$, we have $(b * x) * x=x * x$.

By $\left(P_{3}\right)$, we have $(b * x) * x=0$, so $\underline{R}_{x}^{2}(b)=0$.

Hence $\underline{R}_{x}^{2}(b)=0 \Leftrightarrow \underline{L}_{b}(x)=x, \forall x \in Z$.

Theorem 4.3. Let $\underline{L}_{x}$ be a left map on PU-Algebra $Z$. Then $\underline{L}_{x}^{n}$ is an endomorphism if and only if $\underline{L}_{x}^{n}=\underline{L}_{x}^{n+1}$

Proof. Let $\underline{L}_{x}^{n}$ is an endomorphism then $\forall a \in Z$.

$\underline{L}_{a}^{n+1}=\underline{L}_{x}^{n}\left(\underline{L}_{x}(a)\right)=\underline{L}_{x}^{n}(x * a)=\underline{L}_{x}^{n}(x) * \underline{L}_{x}^{n}(a)=\underline{L}_{x}^{n}(a), \quad \because \underline{L}_{x}^{n}(x)=0$.

Conversely : - If $\underline{L}_{x}^{n}=\underline{L}_{x}^{n+1}$ then clearly we have $\underline{L}_{x}^{n}=\underline{L}_{x}^{n+m}$.

Now from proposition $\left(P_{n}\right)$, we have

$\underline{L}_{x}^{n}(a * b)=a * \underline{L}_{x}^{n}(b)$.

And by $\left(P_{o}\right)$ we have

$\underline{L}_{x}(a) * \underline{L}_{x}(b)=a * b$. Replacing ' $b^{\prime}$ by ${ }^{\prime} \underline{L}_{x}^{n}(b)^{\prime}$ we get

$\underline{L}_{x}^{n}(a) * \underline{L}_{x}^{n}\left(\underline{L}_{x}^{n}(b)\right)=a * \underline{L}_{x}^{n}(b) \Rightarrow \underline{L}_{x}^{n}(a) * \underline{L}_{x}^{n+n}(b)=a * \underline{L}_{x}^{n}(b) \Rightarrow \underline{L}_{x}^{n}(a) * \underline{L}_{x}^{n}(b)=$ $a * \underline{L}_{x}^{n}(b)$.

Comparing equations (1) and (2) we get

$\underline{L}_{x}^{n}(a * b)=\underline{L}_{x}^{n}(a) * \underline{L}_{x}^{n}(b)$.

Hence $\underline{L}_{x}^{n}$ is an endomorphism if and only if $\underline{L}_{x}^{n}=\underline{L}_{x}^{n+1}$.

Theorem 4.4. If $Z$ is a PU-algebra then $\underline{R}_{a * b}=\underline{L}_{a} \circ \underline{R}_{b}, \forall a, b \in Z$.

Proof. $\underline{R}_{a * b}(c)=c *(a * b)$. From proposition $\left(P_{5}\right)$, we have

$\underline{R}_{a * b}(c)=a *(c * b)=\underline{L}_{a}(c * b)=\underline{L}_{a}\left(\underline{R}_{b}(c)\right)=\left(\underline{L}_{a} \circ \underline{R}_{b}\right)(c), \forall c \epsilon Z$.

Hence $\underline{R}_{a * b}=\underline{L}_{a} \circ \underline{R}_{b}, \forall a, b \in Z$.

Theorem 4.5. If $(Z, *, 0)$, is a PU-Algebra and $a * b=0$, then $\underline{L}_{a} \circ \underline{R}_{b}=\underline{R}_{o}, \forall a, b \in Z$.

Proof. Since $a * b=0$. Now consider $\left(\underline{L}_{a} \circ \underline{R}_{b}\right)(c)=\underline{L}_{a}\left(\underline{R}_{b}(c)\right), \forall$ c $\epsilon Z$.

So $\left(\underline{L}_{a} \circ \underline{R}_{b}\right)(c)=\underline{L}_{a}(c * b)=a *(c * b)$. From proposition $\left(P_{5}\right)$, we have

$\left(\underline{L}_{a} \circ \underline{R}_{b}\right)(c)=c *(a * b)=c * 0$, so $\left(\underline{L}_{a} \circ \underline{R}_{b}\right)(c)=\underline{R}_{o}(c) . \quad \because a * b=0$.

Theorem 4.6. If $(Z, *, 0)$, is a PU-Algebra then for all $x \epsilon Z$ the $\operatorname{Ker}\left(\underline{R}_{x}^{2}\right)$ is a subalgebra and ideal of $Z$.

Proof. Let $a * b \in \operatorname{Ker}\left(\underline{R}_{x}^{2}\right)$, then $\underline{R}_{x}^{2}(a)=0$ and $\underline{R}_{x}^{2}(b)=0$. From proposition $\left(P_{d}\right), \underline{R}_{x}^{2}$ is an endomorphism therefore for any $a, b \in Z$, we have $\underline{R}_{x}^{2}(a * b)=\underline{R}_{x}^{2}(a) * \underline{R}_{x}^{2}(b)=0 * 0=0$, so $a * b \epsilon \operatorname{Ker}\left(\underline{R}_{x}^{2}\right)$. Hence $\operatorname{Ker}\left(\underline{R}_{x}^{2}\right)$ is a subalgebra.

Next we prove the second part of the theorem.

$\underline{R}_{x}^{2}(0)=(0 * x) * x=x * x=0 \Rightarrow 0 \epsilon \operatorname{Ker}\left(\underline{R}_{x}^{2}\right)$, so first condition of the definition 2.6 holds in $\operatorname{Ker}\left(\underline{R}_{x}^{2}\right)$. Now consider if $a, a * b \epsilon \operatorname{Ker}\left(\underline{R}_{x}^{2}\right)$, then we have $\underline{R}_{x}^{2}(a)=0$, and $\underline{R}_{x}^{2}(a * b)=0$. From proposition $\left(P_{d}\right), \underline{R}_{x}^{2}$ is an endomorphism therefore we have $\underline{R}_{x}^{2}(a) * \underline{R}_{x}^{2}(b)=0, \Rightarrow 0 * \underline{R}_{x}^{2}(b)=0$. From proposition $\left(P_{1}\right)$, we have $\underline{R}_{x}^{2}(b)=0$, so $b \epsilon \operatorname{Ker}\left(\underline{R}_{x}^{2}\right)$. Thus second condition of definition of 2.6 also holds in $\operatorname{Ker}\left(\underline{R}_{x}^{2}\right)$. 
Hence $\operatorname{Ker}\left(\underline{R}_{x}^{2}\right)$, is an ideal of $\mathbf{Z}$.

Theorem 4.7. If $(Z, *, 0)$, is a PU-Algebra then for all $x \in Z$ the $\mathbf{F i x}\left(\underline{R}_{x}^{2}\right)$, is a subalgebra and ideal of $Z$.

Proof. Let $a, b \in \mathbf{F i x}\left(\underline{R}_{x}^{2}\right)$, then $\underline{R}_{x}^{2}(a)=a$, and $\underline{R}_{x}^{2}(b)=b$. From proposition $\left(P_{d}\right), \underline{R}_{x}^{2}$ is an endomorphism therefore for any $a, b \in Z$, we have $\underline{R}_{x}^{2}(a * b)=\underline{R}_{x}^{2}(a) * \underline{R}_{x}^{2}(b)=a * b$, so $a * b \in \mathbf{F i x}\left(\underline{R}_{x}^{2}\right)$. Hence $\mathbf{F i x}\left(\underline{R}_{x}^{2}\right)$, is a subalgebra.

Next we prove the second part of the theorem.

$\underline{R}_{x}^{2}(0)=(0 * x) * x=x * x=0$, so $0 \epsilon \mathbf{F i x}\left(\underline{R}_{x}^{2}\right)$.

So first condition of the definition 2.6 holds in $\mathbf{F i x}\left(\underline{R}_{x}^{2}\right)$.

Now consider if $a, a * b \epsilon \mathbf{F i x}\left(\underline{R}_{x}^{2}\right)$, then we have $\underline{R}_{x}^{2}(a)=a$ and $\underline{R}_{x}^{2}(a * b)=a * b$. From proposition $\left(P_{d}\right), \underline{R}_{x}^{2}$ is an endomorphism therefore for we have $\underline{R}_{x}^{2}(a) * \underline{R}_{x}^{2}(b)=$ $a * b$, so $a * \underline{R}_{x}^{2}(b)=a * b$. From proposition $\left(P_{13}\right)$, we have $\underline{R}_{x}^{2}(b)=b \Rightarrow b \epsilon \mathbf{F i x}\left(\underline{R}_{x}^{2}\right)$. Thus second condition of the definition 2.6 holds in $\mathbf{F i x}\left(\underline{R}_{x}^{2}\right)$. Hence $\mathbf{F i x}\left(\underline{R}_{x}^{2}\right)$, is an ideal of $Z$.

\section{REFERENCES}

[1] Q.P. Hu, On BCH-algebras, In Mathematics Seminar Notes, 11, (1983) 313-320.

[2] Y. Imai and K. Iseki, On axiom systems of propositional calculi.I, Proceedings of the Japan Academy, 41,No.6 (1966) 436-439.

[3] K. Megalai and A.Tamilarasi, Classification of TM-algebra, IJCA Special Issue on "Computer Aided Soft Computing Techniques for Imaging and Biomedical Applications"CASCT.

[4] S. M. Mostafa, M. A. Naby and A. I. Elkabany, New view of ideals on PU-algebra, International Journal of Computer Applications.111,No.4 (2015) 1-18.

[5] J. Neggers and H. S .Kim, On d-algebras, Mathematica Slovaca,49,No.1 (1999)19-26.

[6] J. Neggers, S.S.Ahn, and H.S.Kim,On Q-algebras, International Journal of Mathematics and Mathematical Sciences,27,(2001). 